\title{
RFID Technology \& its Application for Detection of Removal of Fish Plate in Rail Tracks
}

\author{
Subhashrahul $\mathbf{S}^{1}$,Pradeep S Kumar ${ }^{2}$ \\ $1^{\text {(ECE dept,KCG college of technology,India) }}$ \\ $2^{\text {(ECE dept,KCG college of technology,India) }}$
}

\begin{abstract}
RFID, or Radio Frequency Identification, is the term used for technologies that leverage radio waves to identify items automatically. Typically, this happens through a stored serial number on the tag that identifies a product. The tag may also have other information, such as where the item was made, manufacture date and other information stored in that item like temperature, vibration, contact status, current, voltage, speed , pressure, flow, etc. RFID has two main components namely RFID reader and RFID tags. A reader ( $R$ FID interrogator) is basically a radio frequency (RF) transmitter and receiver, controlled by a microprocessor or digital signal processor. The reader, using an attached antenna, captures data from tags, then passes the data to a computer for processing. As with tags, readers come in a wide range of sizes and offer different features. Readers can be affixed in a stationary position, portable or even embedded in electronic equipment such as print-on-demand label printers. RFID technology can be used effectively for many applications and one of them is detection of removal of fish plate in advance to avoid any derailment or damage to Locos and wagons. RFID tags can be attached in each fish plate and if there is any removal of fish plate then it can be sensed by RFID reader. RFID reader can read tags upto a distance of one kilometre and thus a RFID reader located in the LOCO can detect any removal of fish plate by sensing its corresponding TAG for one kilometre range.
\end{abstract}

Keywords- Fish plates,Readers,RFID,Tags,Tracks

\section{INTRODUCTION OF RFID SYSTEM}

RFID is a complete system solution that operates in the electromagnetic spectrum to transmit data without contact or line of sight. It is an automatic identification and data collection technology utilizing "electronic" programmable tags for tracking, tracing and identification of objects. RFID enables identification from a distance, and unlike the bar-code technology, it does so without requiring a line of sight . RFID tags (see figure 1) support a larger set of unique IDs than bar codes and can incorporate additional data such as manufacturer, product type, and even measure sensitive parameters such as temperature, relative humidity, vibration, etc. Furthermore, RFID systems can discern many different tags located in the same area.

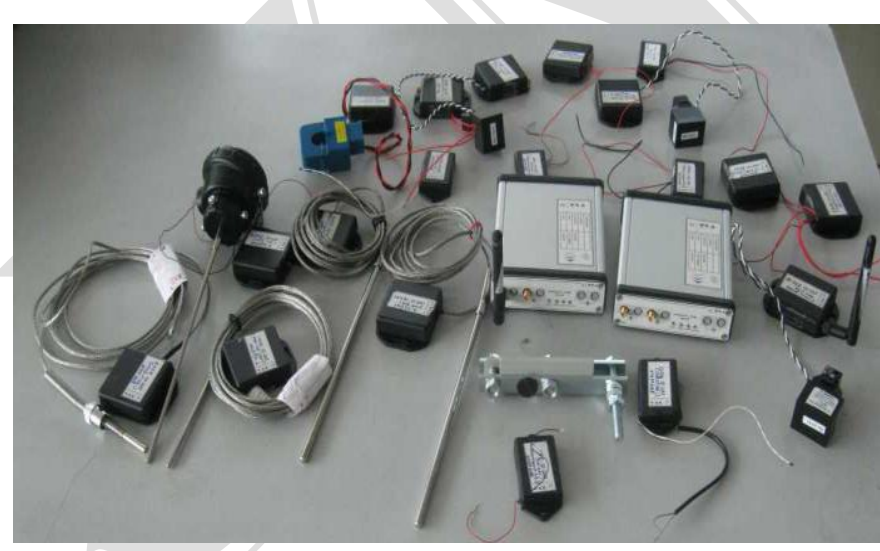

Fig. 1 RFID readers \& different types of RFID tags

RFID is a system with the following components:

- RFID Tag (Transponder)

- RFID Reader (Interrogator)

- Antenna

- Computer System

\subsection{RFID Tag (Transponder)}

RFID tags consist of an integrated circuit (IC) attached to an antenna-typically a small coil of wires-plus some protective packaging (like a plastic card) as determined by the application requirements. Tags also sometimes are called "transponders". RFID tags can come in many forms and sizes. Some can be as small as a grain of rice. Data is stored in the IC and transmitted through the antenna to a reader. RFID tags are either "passive" (no battery) or "active" (self-powered by a battery).

Another distinguishing feature of modern RFID is that tags can contain far more information than a simple ID. They can incorporate additional read only or read-write memory, which a reader can then further interact with. Read-only memory might contain additional product details that may not 


\section{Subhashrahul S, Pradeep S Kumar / IOSR Journal of Engineering (IOSRJEN) www.iosrjen.org

Vol. 2 Issue 2, Feb.2012, pp. 229-233

to be read every time a tag is interrogated but are available when required. For example, the tag memory might contain a batch code, so if some products are found to be faulty, the code can help to find other items with the same defects. Tag memory can also be used to enable tags to store selfdescribing information. Although a tag's unique ID can be used to recover its records in an online database, communication with the database might not always be possible. For example, if a package is misdirected during transportation, the receiving organization might not be able to determine its correct destination. Additional destination information written into the tag would obviate the need and cost of a fully networked tracking system.

Other RFID applications take advantage of read-write memory available in some tag types. Although the size of these memories is currently small but it is likely to grow in the future and to be used in creative ways. These tags could lead to a distributed memory capability embedded in our surroundings. If locations in a city were tagged with RFID, a reader could write messages directly into the tag. This might be used for historical data or for updates about nearby services. Time stamps can also be stored in an RFID memory alongside other data that has been written there. Unfortunately, passive RFID tags do not have the continuous power needed to support an onboard clock, so time stamps could not be derived from the tag itself. However, the readers-powered from the infrastructure or from batteries in a handheld unit - could contain an electronic clock and write time stamps alongside other data written into the tag.

\subsection{RFID Reader (Interrogator)}

The reader/interrogators can differ quite considerably in complexity, depending upon the type of tags being supported and the functions to be fulfilled. However, the overall function is to provide the means of communicating with the tags and facilitating data transfer. Functions performed by the reader may include quite sophisticated signal conditioning, parity error checking and correction. Once the signal from a transponder has been correctly received and decoded, algorithms may be applied to decide whether the signal is a repeat transmission, and may then instruct the transponder to 733 cease transmitting. This is known as the "Command Response Protocol" and is used to circumvent the problem of reading multiple tags in a short space of time. Using interrogators in this way is sometimes referred to as "Hands Down Polling". An alternative, more secure, but slower tag polling technique is called "Hands Up Polling" which involves the interrogator looking for tags with specific identities, and interrogating them in turn. This is contention management, and a variety of techniques have been developed to improve the process of batch reading. A further approach may use multiple readers, multiplexed into one interrogator, but with attendant increases in costs. Readers can be fixed, i.e. mounted, or portable such as a handheld depending on the application. Fig. 2 shows a typical RFID Reader with all communication features.

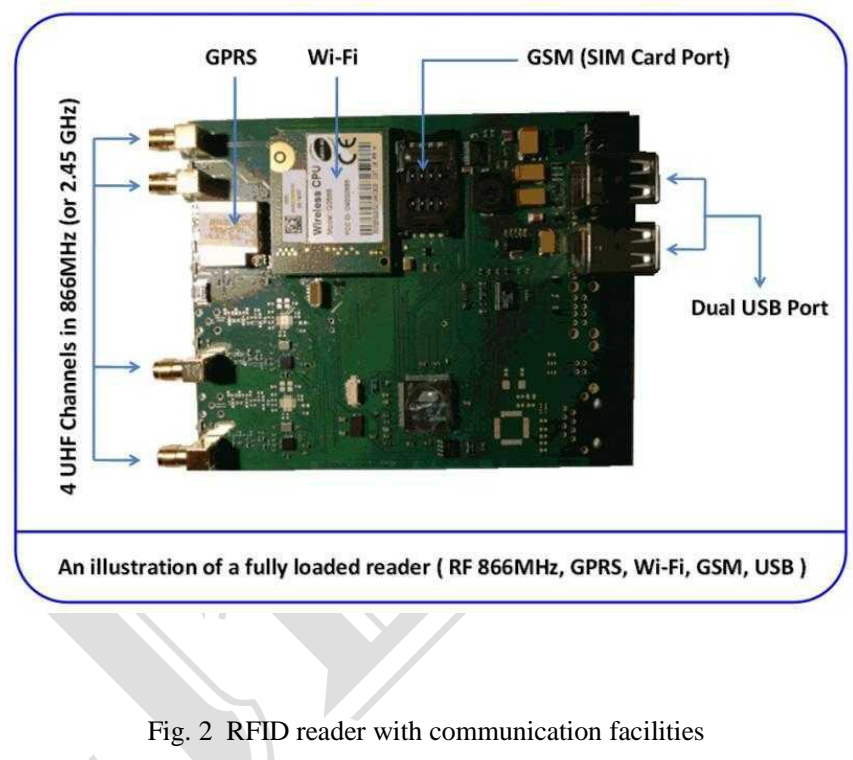

\subsection{Antenna}

A typical reader contains an antenna to transmit information to the tag as well as receive it from the tag. The size and form of the antenna will be dependent on the specific application as well as frequency chosen. It typically houses a decoder and RF module as well as the antenna. Similarly, tags also have antenna for its communication with Readers. The tag antenna is the means by which the device senses the interrogating field and, where appropriate, the programming field and also serves as the means of transmitting the tag response to readers.

\subsection{Computer System}

An RFID system can be stand-alone or interfaced to an IT platform for exchange of information. In either situation, a host system is needed to collect the data to convert into useful information for the end-user. Information is sent to and read from RFID tags by a reader using radio waves. In passive systems, which are the most common, an RFID reader transmits an energy field that "wakes up" the tag and provides the power for the tag to respond to the reader. In active systems, a battery in the tag is 734 used to boost the effective operating range of the tag and to support additional features over passive tags, such as temperature sensing. Data collected from tags is then passed through communication interfaces (cable or wireless) to host computer systems in the computer systems for interpretation, storage, and action. An RFID 


\section{Subhashrahul S, Pradeep S Kumar / IOSR Journal of Engineering (IOSRJEN) www.iosrjen.org \\ ISSN : 2250-3021}

Vol. 2 Issue 2, Feb.2012, pp. 229-233

reader can communicate with a computer by variety of interfaces like RS232, USB, Ethernet, Bluetooth and GPRS. Fig. 3 shows an RFID reader with connected computer system for monitoring tags data.

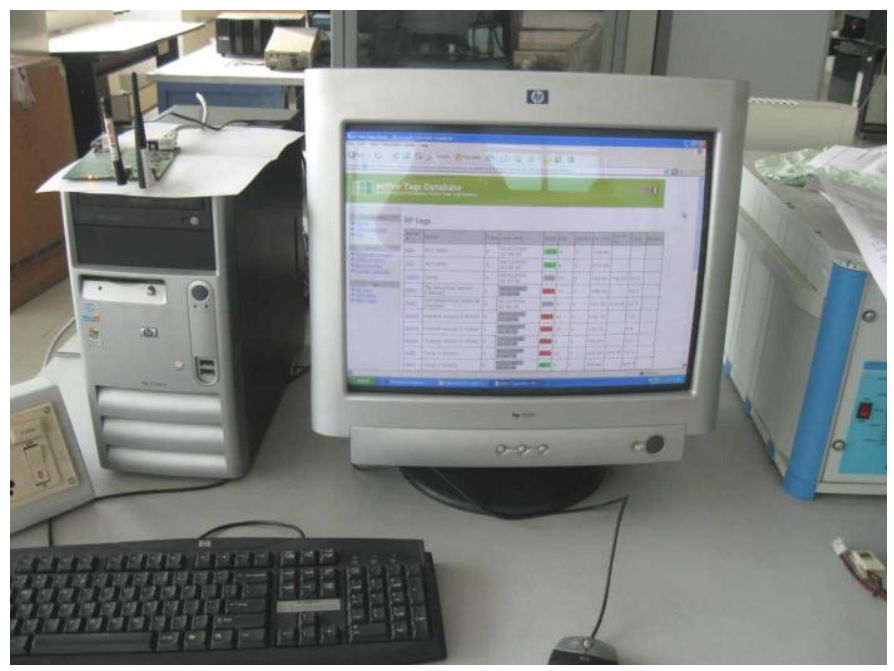

Fig. 3 RFID Reader with required software in computer for data acquisition

\section{WORKING PRINCIPLE OF RFID SYSTEM}

Communication of data between tags and a reader is by wireless communication. Two methods distinguish and categorise RFID systems, one based upon close proximity electromagnetic or inductive coupling and one based upon propagating electromagnetic waves. Coupling is via 'antenna' structures forming an integral feature in both tags and readers. While the term antenna is generally considered more appropriate for propagating systems it is also loosely applied to inductive systems. Transmitting data is subject to the vagaries and influences of the media or channels through which the data has to pass, including the air interface. Noise, interference and distortion are the sources of data corruption that arise in practical communication channels that must be guarded against in seeking to achieve error free data recovery. Moreover, the nature of the data communication processes, being asynchronous in nature, requires attention to the form in which the data is communicated. Structuring the bit stream to accommodate these needs is often referred to as channel encoding 735 and although transparent to the user of an RFID system the coding scheme applied appears in system specifications. Various encoding schemes can be distinguished, each exhibiting different performance features. To transfer data efficiently via the air interface or space that separates the two communicating components requires the data to be superimposed upon a rhythmically varying (sinusoidal) field or carrier wave. This process of superimposition is referred to as modulation, and various schemes are available for these purposes, each having particular attributes that favour their use. They are essentially based upon changing the value of one of the primary features of an alternating sinusoidal source, its amplitude, frequency or phase in accordance with the data carrying bit stream. On this basis one can distinguish amplitude shift keying (ASK), frequency shift keying (FSK) and phase shift keying (PSK). In addition to non-contact data transfer, wireless communication can also allow non-line-of-sight communication. However, with very high frequency systems more directionality is evident and can be tailored to needs through appropriate antenna design. The physical wiring constraints allow communication links and networks to be effectively isolated from each other in a wired communication system. The approach that is generally adopted for radio frequency communication channels is to separate them on the basis of frequency allocation.

Three frequency ranges are generally distinguished for RFID systems, low, intermediate (medium) and high. The following table (Table-1) summarises these three frequency ranges, along with the typical system characteristics and examples of major areas of application.

TABLE 1

\section{COMMONLY USED RFID FREQUENCY BANDS AND} APPLICATIONS

\begin{tabular}{|l|l|l|}
\hline \multicolumn{1}{|c|}{$\begin{array}{c}\text { Frequency } \\
\text { Band }\end{array}$} & \multicolumn{1}{c|}{ Characteristics } & \multicolumn{1}{c|}{$\begin{array}{c}\text { Typical } \\
\text { Applications }\end{array}$} \\
\hline $\begin{array}{l}\text { Low } \\
100-500 \mathrm{kHz}\end{array}$ & $\begin{array}{l}\text { Short to medium read } \\
\text { range Inexpensive } \\
\text { low reading speed }\end{array}$ & $\begin{array}{l}\text { Access control } \\
\text { Animal identification } \\
\text { Inventory control }\end{array}$ \\
\hline $\begin{array}{l}\text { Intermediate } \\
10-15 \mathrm{MHz}\end{array}$ & $\begin{array}{l}\text { Short to medium read } \\
\text { Range potentially } \\
\text { inexpensive medium } \\
\text { reading speed }\end{array}$ & $\begin{array}{l}\text { Access control } \\
\text { Smart cards }\end{array}$ \\
\hline $\begin{array}{l}\text { High } \\
850-950 \mathrm{MHz} \\
2.4-5.8 \mathrm{GHz}\end{array}$ & $\begin{array}{l}\text { Long read range } \\
\text { High reading speed } \\
\text { Expensive }\end{array}$ & $\begin{array}{l}\text { Railroad car } \\
\text { monitoring Fish plate } \\
\text { monitoring Toll } \\
\text { collection systems } \\
\text { Industrial } \\
\text { applications for } \\
\text { condition monitoring }\end{array}$ \\
\hline
\end{tabular}

\section{ACTIVE RFID FOR INDUSTRIAL APPLICATIONS}

Active RFID is a technology not too dissimilar in concept to the passive RFID systems. Wireless tags automatically identify and account for objects they are attached with. In passive tagging, small and low-cost tags are attached to objects and 736 read via a reader infrastructure. 


\section{Subhashrahul S, Pradeep S Kumar / IOSR Journal of Engineering (IOSRJEN) www.iosrjen.org \\ ISSN : 2250-3021}

Vol. 2 Issue 2, Feb.2012, pp. 229-233

Passive RFID systems run the risk of poor accuracy due to an unacceptably low tag signal strength and poor reliability from signal interference. On the other hand, active RFID tags have batteries on-board enabling them to be recognized over greater distances when placed on many different objects and therefore offer the enterprise a dramatically broad, diverse set of solutions. Active RFID systems tag people, assets and vehicles, as well as deliver sensor data from in and around the enterprise. They are used for identifying, locating, tracking, monitoring, securing and sensing enterprise objects. The tags can be programmed to emit beacon signals at a particular frequency. Greater distances are achieved outdoors as in the case of fish plate removal detection system. The choice of tag type, size, function and receiver placement is based on the solution to be architected. The variations are endless and initial active tag systems form a platform for scalability and for adding new applications as needed.

A variant of active RFID technology is "Dual-Active" RFID tagging, or activating tags to transmit their signal only when needed. Individual Radio Frequency (RF) activation fields are established at control points for the purpose of activating tags to transmit their location. With dual-active RFID, tags are automatically triggered whether on equipment or personnel, and offer reliable operations under virtually any setting. The robust long range signal from active RFID combined with dual-active location monitoring capabilities are solving problems that are not possible with other wireless technology.

In the sensor area, active tags can have sensors embedded inside the tag or sensor inputs can be connected from outside. Temperature, contact, voltage, current, vibration and humidity can be sensor inputs, useful for many industries. Any two-wire digital sensor output can be connected into a tag to trigger an alarm condition. This means the sensor implementations are highly flexible and limitless. Inboard motion sensors can sense the slightest movement or vibration of equipment or assets designed to be stationary and stable. Anti-tamper circuits sense any kind of tampering with the tag, immediately sending an alarm signal.

Passive RFID systems can be used in any power plant for asset management system as well as for store inventory system. It can also be used effectively for power plant maintenance. However, active RFID systems can be used for on-line condition monitoring of any power plant equipment. Active RFID tags connected with temperature, vibration, contact, current, voltage or any other sensors can monitor the health/condition of any power plant equipment. This information can be used to finalise operation and maintenance strategy. RFID systems are more useful in power plant areas where other conventional measurement systems are not available and operation and maintenance engineers feel that information regarding these areas may be useful for operation $\&$ maintenance decisions.

\section{DETECTION OF FISH PLATE REMOVAL IN RAIL TRACKS}

One major issue is availability of coals from nearby coal mines. Generally, coals are transported from coal mines to plants using railway tracks. There are many cases of 737 fish plate's removal and this had caused derailment of locos \& wagons. These derailment / accident of locos \& wagons caused delay in coal transportation. Sometimes, these accidents / derailment can also damage locos \& wagons beyond repair.

RFID technology can be used effectively for detection of removal of fish plate in advance to avoid any derailment or damage to Locos and wagons. RFID tags can be attached in each fish plate and if there is any removal of fish plate then it can be sensed by RFID reader. RFID reader can read tags upto a distance of one kilometre and thus a RFID reader located in the LOCO can detect any removal of fish plate by sensing its corresponding TAG in one kilometre range.

This concept has been demonstrated successfully at Loco maintenance bay. RFID reader was kept inside the Loco and RFID tags were kept on rail tracks. Any contact status change in any RFID tags can be sensed by the RFID reader inside the Loco. A simple contact switch (sensor) was incorporated into RFID tags, with a thin loop of wire extending from the tag through the fish plate and back to the tag. If any tampering occurs in the fish plate, the wire breaks and this can be sensed by any RFID reader inside any Loco. Fig. 4 shows an arrangement of RFID sensors in fish plate \& RFID reader in a Loco.

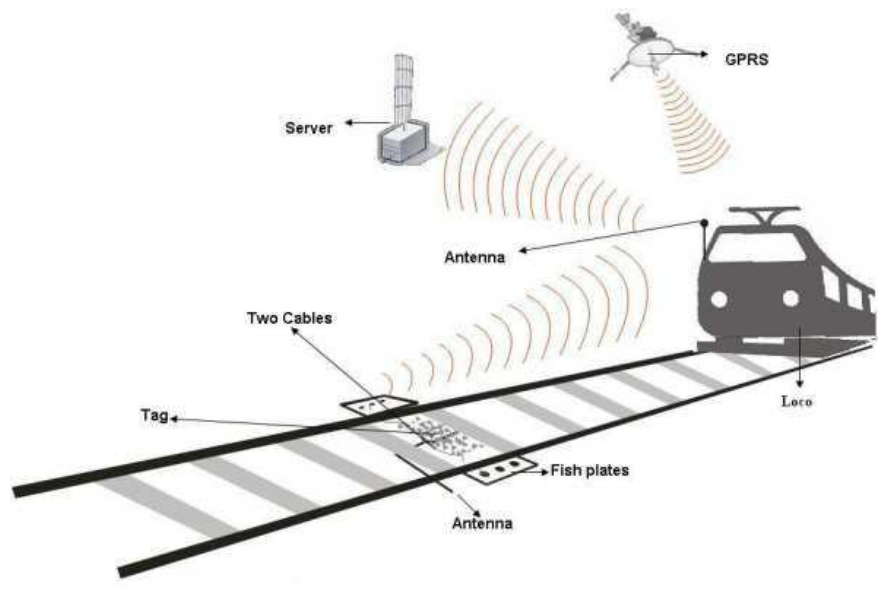

Fig. 4 - RFID tag connected in Fish plate joints \& RFID reader in Loco 


\section{Subhashrahul S, Pradeep S Kumar / IOSR Journal of Engineering (IOSRJEN) \\ www.iosrjen.org \\ ISSN : 2250-3021}

Vol. 2 Issue 2, Feb.2012, pp. 229-233

\section{CONCLUSION}

RFID is a viable technology with many applications that are well suited to its functionality. The future for RFID is strong as evidenced by its continued growth in the past several years. Based on the momentum of RFID and the breadth of the applications across multiple industries, there is no doubt that RFID will grow rapidly and become pervasive throughout the enterprise. In fact, with system costs declining and business opportunities exploding, one can envision a day when every person and every asset in the enterprise is tagged.

Although there are issues of cost of the complete RFID system \& safety of RFID tags on rail tracks but this solution seems to be technically viable. If size of RFID tags can be reduced then it can be kept easily inside the railway sleepers. Fish plate joints can also be reduced by directly welding railway tracks wherever possible. Reduction in fish plate joints will automatically reduce requirement of RFID tags and thus cost of total system will come down. In addition to fish plate removal detection, there are many RFID applications. RFID's potential benefits are large and we are sure to see many novel applications in the future - some of which we can not even begin to imagine.

\section{REFERENCES}

1. K. Finkelzeller, The RFID Handbook, 2nd ed., John Wiley \& Sons, 2003.

2. R. Want, "Enabling Ubiquitous Sensing with RFID," Computer, vol. 37, no. 4, 2004, pp. 84-86.

3. D.W. Engels and S.E. Sarma, "The Reader Collision Problem," white paper MITAUTOID- WH-007, Auto-ID Center, Nov.2001.

4. R. Want, "Introduction to RFID technology," IEEE trans. on pervasive computing, Jan-Mar'2006, pp25-33.

5. Allen Gribenow, "Dual Active Radio Frequency Identification is ideal for Integrator," Axcess White Paper.

6. Zebra's RFID readiness guide: "Ensuring a successful RFID implementation.'

7. "12 Basic steps for DoD RFID Compliance" An Avery Dennison White Paper.

8. "Shrouds of Time - The history of RFID" An AIM Publication.

9. "RFID - A Basic Primer" An AIM Publication. 